\title{
The Analysis of the Barred Galaxy NGC 6701 ${ }^{1}$
}

\author{
I. Márquez ${ }^{2,3}$ \\ Institut d'Astrophysique, 98 bis Bd Arago, 7501/4 Paris (France)
}

\section{Observations}

Images: $1.5 \mathrm{~m}$ Spanish telescope in Calar Alto, Thomson blue coated $1024 \times 1024$ CCD, 0.31" pixel $^{-1}$. Exposition times of 1800, 1500 and $1200(B V R)$ and 2000 seconds ( $\mathrm{H} \alpha$ and continuum) were used. Seeing FWHM $=1^{\prime \prime}$. Spectra for NGC 6701: $3.5 \mathrm{~m}$ telescope, $\mathrm{PA}=25^{\circ}, 115^{\circ}, 145^{\circ}$ and $175^{\circ}$ ( $\mathrm{H} \beta$ region), $2.2 \mathrm{~m}$ telescope, $\mathrm{PA}=25^{\circ}$ and $115^{\circ}$ ( $\mathrm{H} \alpha$ region). The spectrum along the companion galaxy $\left(\mathrm{PA}=0^{\circ}\right)$ was also obtained with the $2.2 \mathrm{~m}$ telescope.

\section{Johnson $B V R$ Images}

In Figure 1a the bar is clearly seen, extending almost all along an axis very close to the disk minor axis. The spiral arms emerge from the bar, with the arm to the north being the more luminous one. The main spiral structure delineates a pseudo-outer ring when the arms close together. The prominent bar structure has a diamond-like aspect $\left(22^{\prime \prime} \times 12^{\prime \prime}\right)$ mainly traced by the two more intense sides. The zones just immediately inside these linear paths are notably fainter, as expected for a region in which the presence of dust is important. The ellipse fitting to the $\mu_{B}=23.5 \mathrm{mag} \operatorname{arcsec}^{-2}$ isophote gives $\mathrm{a}=1$ ? $3, \mathrm{~b}=1$ ! 2 and $\mathrm{PA}=$ $25^{\circ}$. Total (corrected) magnitudes are $m_{B}=12.96(12.65), m_{V}=12.44(12.20)$ and $\mathrm{m}_{I}=11.64(11.46)$.

The linear luminous paths along two of the diamond sides show bluer colors, and appear clearly traced over the background red bar. The colors are the bluest for the knots on the ends of the diamond, similar to those corresponding to the knots in the west arm. There is a very red non-complete ring-like structure to the galactic center.

The bar contamination on the isophotal profile is not negligible, even for the outermost regions. Consequently, the obtained components should be considered as merely indicative; $\mathrm{L}_{b a r}+\mathrm{L}_{\text {ring }}=3 \% \mathrm{~L}_{\text {total }}$ and $\mathrm{L}_{b u l g e} / \mathrm{L}_{\text {disk }}=0.36$.

\footnotetext{
${ }^{1}$ Based on data obtained at the $1.5 \mathrm{~m}$ telescope of the Estación de Observación de Calar Alto, Institnto Geográfico Nacional and the Consejo Superior de Investigaciones Científicas through the Instituto de Astrofísica de Andalucía

${ }^{2}$ Visiting Astronomer, German-Spanish Astronomical Center, Calar Alto, operated by the MaxPlanck-Institut fur Astronomie jointly with the Spanish National Commission for Astronomy

${ }^{3}$ Instituto de Astrofísica de Andalucía (C.S.I.C.), Apdo 3004, 18080 Granada (Spain)
} 

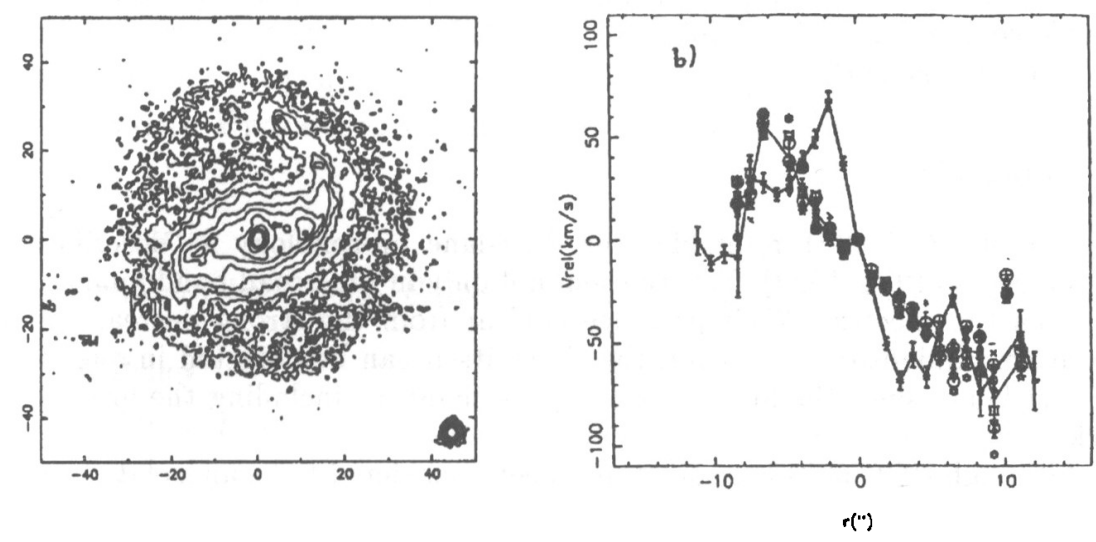

Figure 1. (a) Isophotal $V$ image. (b) Rotation curve given by the gas (solid line) and the stars (big points).

\section{3. $\mathrm{H} \alpha$ image}

Some diffuse emission can be seen along the bar and in the spiral arm to the north. The maximum is reached at the galactic center, showing another bright knot just in the vertices of the diamond-like bar structure. There are some features that can not be delineated in $B$, as the arm to the south, corresponding to very recent star forming regions. The total flux is $\log \left(\mathrm{F}_{H \alpha}\right)=-11.08$, to which the central region contributes $9 \%$.

\section{Spectroscopy}

From the different spectra, $c z=3950 \pm 10 \mathrm{~km} \mathrm{~s}^{-1}$, in agreement with the value given by $\mathrm{RC} 3$, within the errors. The rotation curve is given in Figure $1 \mathrm{~b}$. The inner gradient amounts to $171 \mathrm{~km} \mathrm{~s}^{-1} \mathrm{kpc}^{-1}$ which lies in the lower end of the range occupied by Sa galaxies. From the spectrum along PA = $175^{\circ}$ we determine $V_{c o r r}=153 \mathrm{~km} \mathrm{~s}^{-1}$ for a distance of $r=19^{\prime \prime}=0.41 \times \mathrm{r}_{25}$. The total mass inside $\mathrm{r}_{25}$ is $M=6.8 \times 10^{10} M \odot\left(\mathrm{H}_{o}=75 \mathrm{~km} \mathrm{~s}^{-1} \mathrm{Mpc}^{-1}\right)$. The velocity distribution along the minor axis shows a rotation of maximum amplitude around $60 \mathrm{~km} \mathrm{~s}^{-1}$. Gas and stars are kinematically decoupled (Figure $1 b)$. This effect is interpreted as due to the presence of peculiar motions in the central region. The stellar velocity dispersions have values around 100 and 150 $\mathrm{km} \mathrm{s}^{-1}$.

For $\mathrm{PA}=115^{\circ}$, the slit crosses one of the bar dust lanes; for this region $[\mathrm{NII}] / \mathrm{H} \alpha$ varies from 0.9 to $1.5,[\mathrm{SII}]_{T} / \mathrm{H} \alpha \approx 0.8, \mathrm{~S} 2 / \mathrm{S} 1 \approx 0.9$ to 0.5 and $[\mathrm{OI}] / \mathrm{H} \alpha$ $\approx 0.06$. The $\mathrm{H} \beta$ absorption avoids a reliable determination of the [OIII] $/ \mathrm{H} \beta$ ratio. But, in spite of this, we can say that the physical processes producing the lines in the dust-lane region are a mixing of photoionization by stars and shocks (Osterbrock 1989) in a dust rich zone. 
Gaussian profile fittings to $\mathrm{H} \alpha$, [NII] and [SII] lines of the spectrum for the possible companion (Nilson 1973) give $c z=4078 \pm 14 \mathrm{~km} \mathrm{~s}^{-1}$, reported for the first time in this work.

\section{Conclusions}

The bar of NGC 6701 resembles the dust-lane morphology as described by Athanassoula $(1984,1992)$. We see them not only in the broad band images, but also from the spectra. Their presence is clear from the strong decrease of the continuum luminosity. Moreover, the shock itself can be detected in one of the two dust lanes, since the line ratios imply mechanisms including the presence of shocks.

Although NGC 6701 has sometimes been considered to be an isolated galaxy it is found to have a very close companion at $130 \mathrm{~km} \mathrm{~s}^{-1}$ and $73 \mathrm{Kpc}$ in projected distance, from which the crossing time is $t_{c} \approx 5 \times 10^{8}$ years. On the other side, the age of the bar would be of less than $\approx 10^{9}$ years (Martin \& Roy 1995), taking into account that there are a number of HII regions along the bar, and following the predictions given by numerical simulations (after $\approx 1 \mathrm{Gyr}$, the gas sinks into the center and just a nuclear starburst is found, as for example the $\mathrm{H} \alpha$ morphology of NGC 6951, Marquez \& Moles 1993).

The times scales obtained for the interaction, the enhancement of the star forming activity, and the bar age are all of the same order. These results can be easily interpreted in a scenario in which the gravitational interaction induces both more efficient star forming processes in the disk of the galaxy, and the formation of such a prominent bar as a result of the gravitational torques exerted by the companion, in agreement with similar bars obtained in numerical simulations of interacting galaxies (Noguchi 1990). Once the bar is induced, it evolves as expected in an early spiral, showing the typical morphology with straight dust lanes due to the presence of shocks in the leading sides of the bar, isophote twisting in the center and an inner ring (Márquez et al. 1995).

\section{References}

Athanassoula, E. 1984, Physics Reports, 114, 319

Athanassoula, E. 1992, MNRAS, 259, 345

Márquez, I. \& Moles, M. 1993, AJ, 105, 2090

Márquez, I., Moles, M., \& Masegosa, J. 1995, A\&A (submitted)

Martin, P. \& Roy, J.-R. 1995, ApJ, 445, 161

Nilson, P. 1973, Uppsala General Catalogue of Galaxies

Noguchi, M. 1990 in Paired and Interacting Galaxies, J. W. Sulentic, W. C. Keel, \& C. M. Telesco, NASA-CP3098, 171

Osterbrock, D. E. 1989, Astrophysics of Gaseous Nebulae and Active Galactic Nuclei, Mill Valley University Science Books 